\title{
A Review on Triangle based Techniques in Biometric Gait Recognition
}

\author{
Monika Jhapate \\ M. Tech \\ Radharaman Engineering \\ College, Bhopal
}

\author{
Lalitesh Choudhary \\ Ass. Professor \\ Radharaman Engineering \\ College, Bhopal
}

\author{
Ravi Singh Pippal \\ Ass. Professor \\ Radharaman Engineering \\ College, Bhopal
}

\begin{abstract}
Biometric system is an analysis of unique biological features of human being. The purpose is used for human security and identification. Different conventional biometric (such as face recognition, iris, fingerprint, etc.) methods are used for security and identification purpose, but they can capture only by physical control or at a close distance from record search. Gait on a behavioral biometric has attracted more attention recently because it can capture at a distance with requiring the earlier consent of the observed object. This survey paper covers the current trends and method of Gait based surveillance system using triangle methods and compare them.
\end{abstract}

\section{Keywords}

Biometric, Gait Recognition, Image Processing, Triangle methods, Pattern Recognition.

\section{INTRODUCTION}

As the world is getting advanced and computerized so the security system which were earlier human controlled [1] are being replaced by a computerized surveillance system. Which is based on image processing. It is used in this system to identify the unique physical property that means Biometric property of a person, Biomatric characterized into two portion physiological properties (face, fingerprint, iris, DNA) and behavioral property (signature, voice, walking pattern).

Previously, biometric research concentrated on human authentication and authorization, utilizations face images, fingerprint, palm prints, shoe print, iris, images and handwriting. But these conventional biometric resources suffer from several limitations such as distance between the camera /scanner and people, people (user) co-operation will consider for authentication and authorization.

For visual surveillance applications, the conventional biometrics resources are difficult to utilize and gait provides in an interesting way. A gait describes the manner of a person's walking i.e. walking pattern recognition. It can be acquired at a same distance and it is necessary without the walker's co-operation or knowledge that's why this method represents as a further security system. One of the methods which are used to make such examination is gait. It can be done by Genetic Algorithms (GA), Artificial Neural Network (ANN), and mathematical concepts (geometric) by using Gabor system. In a previous study [2] the body is divided into two parts the static or fix (upper part) and dynamic or more movable then upper part (Lower part). The upper body part is subdivided into three parts, the first part is the head, the second part is arm the third part is the chest and the lower body part is subdivided into 4 parts, the first part is thigh, which includes hip, second part is the front leg, the third part is back leg and the fourth part is feet. Gait has mainly worked done in the lower body part because the lower portion of the body moves more than upper part so study of moving parts is easy. The front-leg and back-leg are included as separate parts because of the bipedal (cycle) walking style. When a person walks the left leg and the right leg come to front/back by turns and create a cycle.

This survey paper is divided into five sections, one contains an introduction, the second contains an overview of biometric recognition system, section three contains literature contents studied, section four contains comparisons of the triangle techniques based on studied in literature survey and section five contains the conclusion.

\section{OVERVIEW}

An informative survey of the current analysis techniques to data regarding human movement has been outlined by Gavrlla [3], In his work has done visual analysis, looking at gestures and whole body movement. His survey gives results to recognize human and their activities by computer to interact intelligently and effortlessly with a human inhabited environment.

In this basic biometrics surveillance system has following component:

Capture video: The video is captured by high quality digital cameras.

Convert into frames: Videos are converted into various gait frames in one cycle, according view here side view.

Analysis of each frame based on the approach: Each frame is analyzed depends upon the method which is used.

Correlate as a Triangle Feature: In this component new data are stored in the database and if existing values are found, then result is decided.

Correlate as a Triangle Feature: In this component new data are stored in the database and if existing values are found, then result is decided.

Database: Here the data are stored.

Result: Based on input, output is generated. 


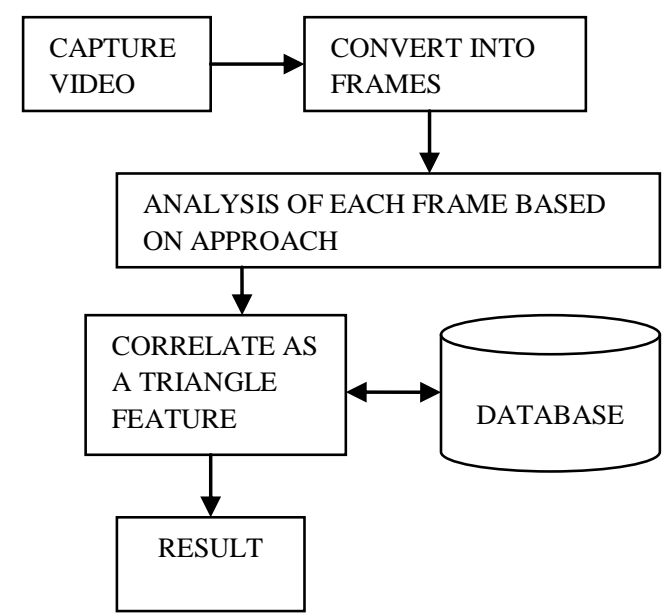

Fig.1 Basic biometrics surveillance system for triangle approach

\section{LITERATURE SURVEY}

In this section we are discussing an approach with different fields. Positioning body joints based approach, Angels based gait detection approach, area of triangle based approach, A Novel Method of Gait based recognition Using Fuzzy Inference, System Gait Geometric Characteristic and Fuzzy Logic based approach.

\subsection{Position joint base human body detection:}

In [4] gait recognition means identify individual persons or subject by analysis of patterns generated in each frame of cycles. Gait recognition is to identify individuals by the way people walk in no consideration of the disturbance such as background, clothes and so on. In the view of biomechanics, the walking of people includes the synchronous movements of hundreds of muscles and joints. Gait is completely determined by the structure of muscle bones. By which all people's movements are based on biped patterns, the gait of each one is different in relative time, step range and so on. So gait is believed to be particular for everyone and can be used as a feature in personal identity recognition, especially in the distance. In this paper they used simple gait recognition method which is based on different points on body joints. In this work they first extract the human or subject frames from of moving bodies in the form of silhouetted images from a given image. Silhouetted means the black or carbon images contain only black image [4]. In that image, 12 different body points identified by them and compute 9 different angles between those points. The angles are angles between trunk called the trunk angle, angle of left arm, angle of right arm, angle of left forearm, angle of right forearm, angle of left thigh, angle of right thigh, angle of left shank and finally angle of right shank.

Then they calculated limb angle. After that, made discrete Fourier transform for each cycle. Two different frequencies, amplitude, frequency and phase frequency of angles are chosen. Finally, apply the nearest neighbor classifier that is used to classify subjects from the database. In their work they used "SOTON" Dataset for simulates their results. The SOTON data set has 118 total images. From those images in their work they used 10 images and give Correct Classification Rate (CCR) 78\%, which had better results than other methods which were presented that time like Body shape and template correlation (CMU) which correct classification rate was $45 \%$, Static body parameters (Georgia Techniques) which CCR was $73 \%$.

\subsection{Angels based gait detection:}

In [5] gait recognition angle based gait detection is important and more efficient than other method. In this work they used two body part of the human as a feature extraction and according to those features of the human body the calculation is done. They had taken three lower parts of the body those all features were from lower because maximum movement is done in lower portion. Here in their work they took both the foot (left feet and right feet) to be more specific they used center point of feet from base as a third party hand which is visible in side view are taken and construct a logical angle. Forgiven angle gait recognition has been considered two features of the human body that is hand and feet for gait recognition is considered. To be more specific center point of base of both feet is taken as vertices of the triangle which will be found using the hand as a third vertex. They calculate [5] the formed angle by the slope method in that method they used tangent formula. They calculated three angles for each frame and after completion of one cycle mean value were calculated.

A cycle is a formed one when a person whose walking [11] posture is being captured reaches to the posture which is same as starting posture of the person. In this paper they focus on angle based analysis and appear the method on CASIA A database in which side view images are given. They have taken some (17) subject images and calculate the angles for each frame for one cycle and after calculation there correct classification rate was $90 \%$ which is more efficient than other methods.

\subsection{An approach for human gait identification based on the area of a triangle:}

In [6] biometric system data to be collected and given as a video input. So, in the preprocessing initial video is captured, and then converted into frames for that particular person. In this work they have considered side [10] view of particular subjects. Here they have considered three parameters of the human body for feature extraction. It is Left hand, right feet and left feet. They consider three feature points. The feature points are taken as a white dot point which represent high resolution. They create a triangle between extracted points. They calculated the length of each edge total three edges are captured [6] edge 1 (a), edge 2 (b) and edge 3 (c) and then for all the frames of one cycle, the mean values of edges are calculated and the stores to those values in database.

Here they calculate correct classification rates for both analyses. The First is for individual distance classification and second is for pair distance correct classification. This experiment demonstrated that a feature selected of a by pair distance gives better result than individual distance. The result shows the correct classification of the first method is $66.6 \%$ and the correct classification of the second method is $82.3 \%$. 
It has been concluded that second analysis they have given better classification rate.

\subsection{A Novel Method of Gait Recognition Using Fuzzy Inference System:}

In [4] this work they used body joint method. Here five different three body parts were taken. The body parts were left feet, right feet and hand. Total five feature points were extracting two points in left feet (toe and ankle) and two in right feet (again toe and ankle). They construct 2 triangles first are between left feet toe, hand and right feet toe and, second between the left foot ankle, the right foot ankle and hand. Here they observed that both triangles were intersecting and two intersecting points generate. They computed those intersection points by parametric line equation. They calculate those points for each frame for each cycle and find the mean value for each cycle and stores in the database. This experiment is all based on the condition of the outdoor gait database environment of various subjects using a side view of the walking direction. After applying the algorithm on this database the correct classification rate is $90 \%$, which result is good as compared to other methods.

\subsection{Gait Recognition with Geometric Characteristic and Fuzzy Logic:}

In [7] the definition of Gait is defined as "A particular way one person walks". It is a process which is divided into stages [8]. Analysis of walking pattern is a gait cycle. The style of walking or gait cycle of every person is unique. [9]. Human gait is the repeated motion of the body parts. Mostly there is no much more changes in head and shoulder motion as compare to hand and legs. The repeated motion part of the body forms a gait cycle. A Gait cycle or stride is defined as a movement when an initial position of a heel comes back again. The single gait cycle is further divided into two phases: In the proposed method two parameters of human bodies have been taken. The First component is hand and another component is feet. The Second parameter is subdivided into two portions it is toe (left and right feet) and (left and right feet). Total five extraction points were identified. The extraction points were decided by high resolution white points. Here two triangles were formed between these five points and those triangles constructed between the toe of left feet, hand and toe of right feet and heel of left leg, hand and heel of right leg. Here two intersection points were taken [7] for study and points are known as I and I'.

The triangle is constructed where point A represent Hand and point $\mathrm{B}, \mathrm{C}$ represents toe of the left feet and toe right feet respectively, point $\mathrm{D}, \mathrm{C}$ represents heel of left feet and heel right feet respectively. In this work they calculated the intersection points for each frame and then calculate for a complete cycle. Then mean values were calculated. These mean values were input of fuzzy inference systems. FIS compare and produces results with the database values according to the following fuzzy rules: Result analysis is done on CASIA dataset for gait recognition of the proposed method. In a proposed work 17 subjects had been taken with 23 frames, which complete the gait cycle, only one side is considered. 17 subjects of MPEG files are converted into JPEG frames, then white dots pixels are inputted on RGB frames of an individual subjects in a proposed gait system after that, these RGB frames are converted into gray scale for further processing, with the database value, if the value is greater than $85 \%$ then matching is excellent, value belongs between 75 to $85 \%$, then matching is good, value lies between 60 to $75 \%$ and matching is average, if value is less than $60 \%$, then matching is poor, these rules are decided by the fuzzy set and the result shows that the correct classification of this method is $88 \%$.

\section{COMPARISON}

After reviewing different papers and work on these approaches, the correct classification rate (CCR) obtained by different researchers in field of triangle based gait can be summarized as following table which results are shown in the table on the basis of their CCR rates and number of people under surveillance.

\begin{tabular}{|c|l|c|c|}
\hline S.No. & \multicolumn{1}{|c|}{ Approach } & $\begin{array}{c}\text { No. of } \\
\text { Persons } \\
\text { under } \\
\text { Surveillance }\end{array}$ & CCR \\
\hline 1. & $\begin{array}{l}\text { Angels based gait } \\
\text { detection }\end{array}$ & 18 & $90 \%$ \\
\hline 2. & $\begin{array}{l}\text { An approach for human } \\
\text { gait identification based } \\
\text { on area of triangle }\end{array}$ & 18 & $82 \%$ \\
\hline 3. & $\begin{array}{l}\text { A Novel Method of } \\
\text { Gait Recognition Using } \\
\text { Fuzzy Inference } \\
\text { System }\end{array}$ & 18 & $90 \%$ \\
\hline 4. & $\begin{array}{l}\text { Gait Recognition with } \\
\text { Geometric } \\
\text { Characteristic and } \\
\text { Fuzzy Logic }\end{array}$ & 15 & $88 \%$ \\
\hline
\end{tabular}

\section{Table 1: Comparison of Various Approaches}

The table 1 indicates that the result of Method based on positioning body joints [4] A Novel Method of Gait Recognition Using Fuzzy Inference System and Angels based gait detection gives the same result which are best result among the above other mentioned methods basically these methods used the concept of fuzzy interface and angle based recognition respectively. At first, they verified the usefulness of the algorithm on the gait database established which includes 18 different subjects. Furthermore the experiment is all based on the condition of side view images. Other methods have also good results, but methods for calculating the area or angle change results. Here we are giving comparison chart between methods and their CCR rate in percentage where $\mathrm{CCR}$ is correct classification rate.

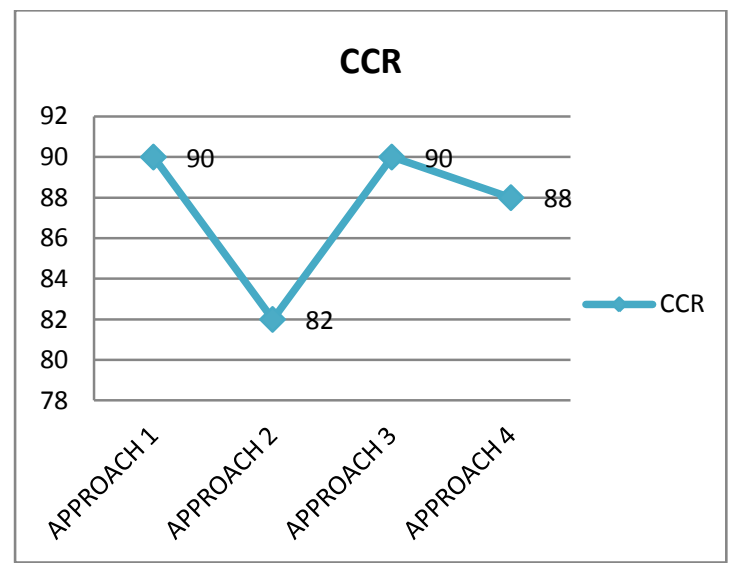

Fig.2: Correct classification rate chart 


\section{CONCLUSION}

In this review paper, we present a comparison between different approaches the techniques which are based on triangle of different body parts. In this review paper, we discussed only lower body part analysis. Here we took those work which is based on common components. All techniques used triangle based method they construct triangle by feature extraction and then recognition is done by various methods. By this review paper, it has been observed that the angle based gait detection and novel method give the same results.

In this review paper they gave information about triangle based technique. These all technique are based on static images. In future we can use these all technique on moveable images.

\section{REFERENCES}

[1] Jyoti Bharti, M. K. Gupta, "Gait Recognition with Geometric Characteristic and Fuzzy Logic", Canadian Journal on Image Processing and Computer Vision Vol. 3 No. 1, March 2012.

[2] Xuelong Li, Stephen J. Maybank,Shuicheng Yan, Dacheng Ta, and Dong Xu,"Gait Components and Their Application to Gender Recognition", IEEE Transactions on Systems, Man, and Cybernetics, Part C Applications and Reviews, vol. 38, no. 2, March 20.

[3] D.M.Gavrila, The visual analysis of human movement: a survey, Computer Vision and Image Understanding, Vol. 73, no.1, January, pp.82-98, 1999.

[4] AI-Hua Wang, ji-Wei Liu, A gait recognition method based on positioning human body joints, proceedings of the 2007 international conference on wavelet analysis and pattern recognition, Beijing, China, 2-4 Nov, 2007.
[5] Arun Kumar Jhapate, Jasvinder Pal Singh, Gait Based Human Recognition System using Single Triangle IJCST Vol. 2, Issue 2, June 2011 ISSN : 2229 - 433 ( Print )| ISSN : 0976-8491 (Online )

[6] Jasvinder Pal Singh, Sanjay Agrawal, An approach for human gait identification based on area, IOSR Journal of Computer Engineering (IOSR-JCE) e-SSN: 2278-0661, p- ISSN: 2278-8727Volume 10, Issue 3 (Mar. - Apr. 2013), PP 43-47.

[7] Mr. Atul Raghuwanshi Mr. Jayesh Surana, A Novel Method of Gait Recognition Using Fuzzy Inference System, International Journal Of Research In Advance Technology In Engineering (IJRATE) Volume 2, Issue 3, June 2014, ISSN: 2347 - 7806.

[8] Nikolaos V. Boulgouris, Dimitrios Hatzinakos, and Konstantinos N. Plataniotis, A challenging signal processing technology for biometric identification, IEEE Signal Processing Magazine,Vol-22, No- 6 pp 78-90, November 2005.

[9] James R. Gage, Peter A. Deluca, Thomas S. Renshaw, Gait Analysis Principles and Applications, The Journal of bone and joint surgery, vol. 77,1607-1623, 1995.

[10] Xuelong Li, Stephen j. Maybank, switching Yan, dancing too, and Dong $\mathrm{Xu}$, gait components and their application to gender recognition, IEEE transactions on systems, man, and cybernetics - part c: applications and reviews, vol. 38 , no. 2 , march 2008 .

[11] Ryuhei Okuno, Satoshi Fujimoto, Jun Akazawa, Masaru Yokoe, Saburo Sakoda and Kenzo Akazawa, 30th Annual International IEEE EMBS Conference Vancouver, British Columbia, Canada, August 20-24, 2008. 